\title{
A Mathematical Tumor Model with Immune Resistance and Drug Therapy: an Optimal Control Approach
}

\author{
L.G. DE PILLIS ${ }^{\mathrm{a}, * \frac{*}{4}}$ and A. RADUNSKAYA ${ }^{\mathrm{b}, \mathrm{t}}$ \\ ${ }^{a}$ Harvey Mudd College, Claremont, CA 91711, and Argonne National Laboratory, Argonne, IL 60439 and ${ }^{b}$ Pomona College, Claremont. \\ CA 91711
}

(Received December, 1999; In final form April 18, 2000)

\begin{abstract}
We present a competition model of cancer tumor growth that includes both the immune system response and drug therapy. This is a four-population model that includes tumor cells, host cells, immune cells, and drug interaction. We analyze the stability of the drug-free equilibria with respect to the immune response in order to look for target basins of attraction. One of our goals was to simulate qualitatively the asynchronous tumor-drug interaction known as "Jeff's phenomenon." The model we develop is successful in generating this asynchronous response behavior. Our other goal was to identify treatment protocols that could improve standard pulsed chemotherapy regimens. Using optimal control theory with constraints and numerical simulations, we obtain new therapy protocols that we then compare with traditional pulsed periodic treatment. The optimal control generated therapies produce larger oscillations in the tumor population over time. However, by the end of the treatment period, total tumor size is smaller than that achieved through traditional pulsed therapy, and the normal cell population suffers nearly no oscillations.
\end{abstract}

Keywords: Cancer, Tumor, Population Models, Competition Models, Mathematical Modeling, Immune System, Optimal Control

\section{INTRODUCTION AND BACKGROUND}

The growth of a cancerous tumor in vivo is a complicated process involving multiple biological interactions. The response of such tumors to active treatment such as chemotherapy and radiotherapy is also complex, but important to understand. Currently, there exists an array of mathematical models of cancer progression and treatment, each of which tends to focus on simulating one or two important elements of the multifaceted process of tumor growth and response to therapy. In a cooperative effort with clinicians and research oncologists, we have been investigating mathematical models of tumor growth with the goal of better understanding how the various aspects of growth and treatment interact with one another. Our investigations led us to develop our own generalized mathematical model of cancer growth, which incorporates several key elements of the growth processes and the effect of their mutual interactions. Addition-

\footnotetext{
* depillis@math.hmc.edu

$\uparrow$ depillis@mcs.anl.gov

\$aradunskaya@pomona.edu
} 
ally, we employ numerical optimal control methods to search for treatment protocols that, in theory, are improvements to the standard protocols in use today.

\subsection{Modeling Tumors}

The development of a cancerous tumor is complex and involves the interaction of many cell types. The tumor itself is not homogeneous; and normal tissue, lymphocytes, macrophages, and other types of cells either grow at the tumor site or are recruited to the tumor through chemotaxis. Cell growth may be stemmed as cells compete for resources and space, but may also be stimulated by the presence of certain cell populations. Through a biochemical process, immunogenic tumor cells and cytotoxic immune cells interact, first binding to form cell conjugates, and then splitting to produce lysed tumor cells, inactivated immune cells, undamaged tumor cells or undamaged immune cells, and debris; see (Kuznetsov et al. 1994) and (Owen and Sherratt 1998). When chemotherapy is administered, a toxic drug is introduced that in principle destroys all cell types to some extent, modifying this interplay among cell populations. Many clinically observed effects are still not well understood in terms of existing models. In this work, we investigate an approach to creating a mathematical model of tumor growth with chemotherapy in which multiple interactions are considered.

As we have noted, much useful work has been done on simplified yet fundamental models involving the interaction between tumor cells and immune cells alone (see (Kuznetsov et al. 1994), (Owen and Sherratt 1998), and (Adam 1993)), between tumor cells and normal cells alone (see (Knolle 1988), (Dibrov et al. 1985), and (Eisen 1979)), and between tumor cells and chemotherapy treatments alone (see (Shochat, Hart, and Agur 1999), (Adam and Panetta 1995), (Martin 1992), (Murray 1990), (Martin et al. 1990), (Swan 1987), (Coldman and Goldie 1986), (Swan 1985), (Dibrov et al. 1985), and (Eisen 1979)).

These models, while extremely useful in providing an understanding of tumor growth and treatment from various perspectives, have not been sufficient to reproduce certain qualitative aspects of interest to the clinicians with whom we are working. To capture some of this elusive qualitative behavior, we have developed a model that incorporates the interactions among tumor cells, normal cells, immune cells, and chemotherapy.

Some work has also been done in the development of stochastic models; see (Castellanos Moreno 1996), (Bartoszyński, Jones, and Klein 1985), (Duc 1985), (Serio 1984), and (Bramson and Griffeath 1980). A stochastic approach can be useful, especially in the context of interactions among populations with low densities. In this work, however, we concentrate on continuous-time deterministic models of tumor growth and treatment. This allows us to apply classical optimal control theory, through which we determine improved chemotherapy administration schedules.

\subsection{Theory versus Observation}

The design of a mathematical model of a biological system is governed by the need to distill the essential behavior of the system and the need to answer specific questions about that system. In our case, our goal was to use the model to design a protocol for chemotherapy that would produce an improved outcome by way of reducing final tumor size without causing large losses in the normal cell population. We also wished to develop a model of tumor growth that would evidence certain clinically observed phenomena brought to our attention by the research oncologists with whom we have been working. The model we have developed, which is built from combining some of the most useful aspects of previously existing models, does in fact exhibit the qualitative behavior we wished to reproduce, including "Jeff's phenomenon" and tumor dormancy.

\subsubsection{Jeff's Phenomenon}

"Jeff's phenomenon" is a clinically observed temporal oscillation in tumor size which is apparently unsynchronized with the administration of chemotherapy. In some patients a tumor continues to grow after treatment, and then, some time after treatment has ceased, 

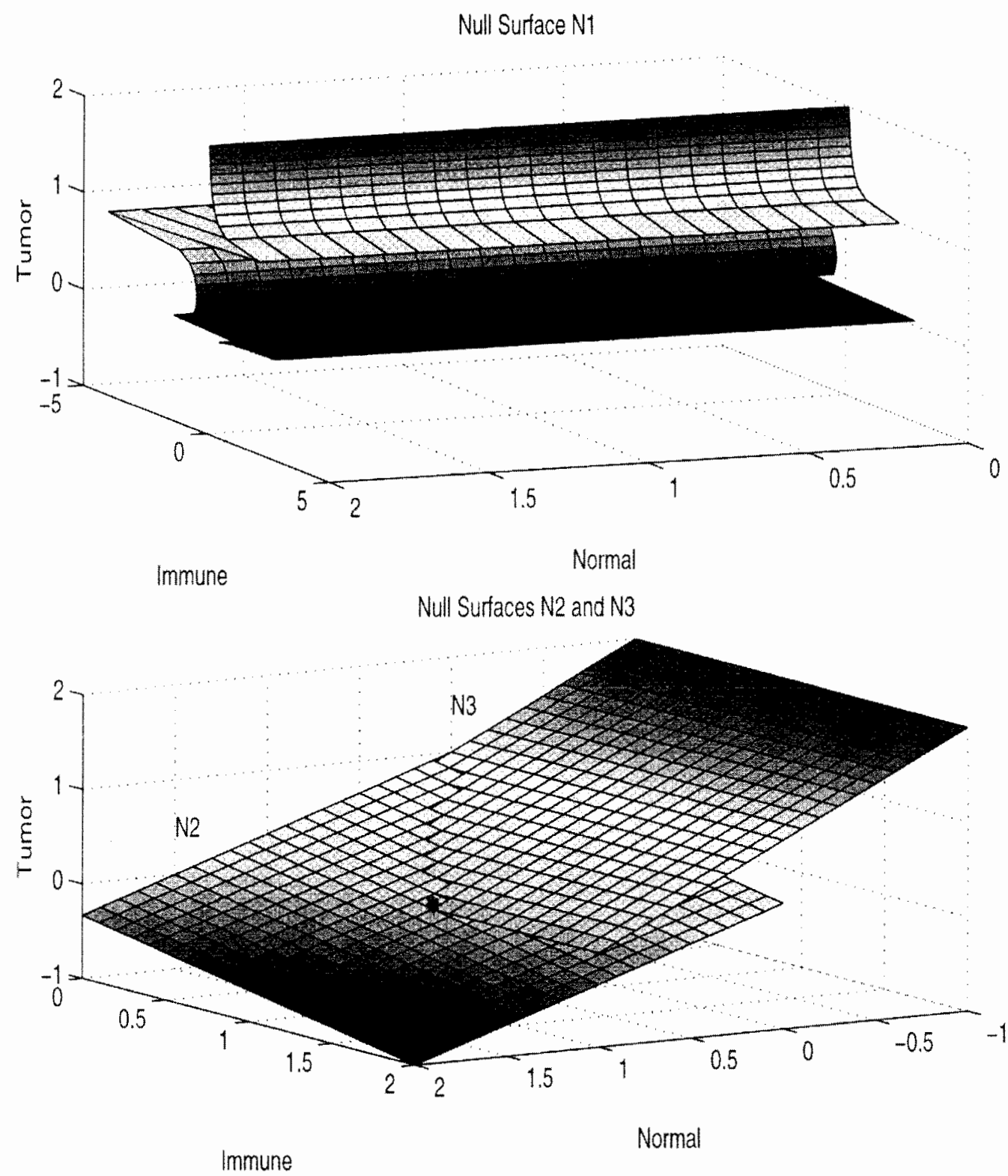

FIGURE 1 The three null-surfaces for the parameter values listed in Section 5.1. The top graph shows the immune null surface N1, which is curved. The bottom graph shows the planar surfaces, N2 and N3, for the tumor and normal rate equations, respectively. The stable coexisting equilibrium for this particular parameter set is marked as a dot on each graph (See Color Plate I at the back of this issue)

begins to decrease in size. According to Thomlinson (1982), these asynchronous responses are not a result of drug resistance, as some may speculate. Therefore, to reflect this asynchronous reaction to cytotoxic drugs, we chose to model the interaction between a drug and the various cells as a continuous-time process rather than an instantaneous kill, as in (Panetta 1996). Thus, the effect of the drug is incorporated into 
the differential equations themselves, rather than occurring as pulsed, instantaneously effective treatments. In our new model the drug affects normal and immune cells, as well as tumor cells. This does, in fact, achieve the desired qualitative effect and causes oscillations in tumor size whose phase and period change over time and are asynchronous with drug administration.

\subsubsection{Tumor Dormancy}

Another phenomenon of current interest to clinicians is tumor dormancy. There is clinical evidence that a tumor mass may disappear, or at least become no longer detectable, and then for no apparent reason may reappear, growing to lethal size. The mechanisms and behavior of this phenomenon have been and continue to be studied from both clinical and mathematical modeling perspectives. Multiple clinical studies document the strong connection between the effects of the immune system and tumor dormancy. For example, Farrar et al. (1999) present the results of clinical studies on murine $B$ cell lymphoma (BCL1) in mice vaccinated with BCL1 Ig. Farrar et al. extend previous work, which demonstrated that $T$ cell-mediated immunity is an important component, in the regulation of tumor dormancy, and demonstrate that CD8 + T cells in particular play a decisive role in both inducing and maintaining a state of tumor dormancy. Again in the context of BCL1 in mice, the work of Morecki et al. (1996) indicates that cell-mediated antitumor immunity contributes to maintenance of the tumor dormant state. In (Matsuzawa et al. 1991b) and (Matsuzawa et al. 1991a), it is shown that Lyt-2+, L3T4- T cells appear to mediate host antitumor immunity to B cell leukemia (DL811) in DDD mice to eradicate leukemic cells and maintain a dormant state. Muller et al. (1998) did a study on tumor dormancy in bone marrow and lymph nodes. Their experiments show that bone marrow and lymph nodes are sites where potentially lethal tumor cells are controlled in a dormant state specifically by the immune system. Stewart (1996) reviewed findings of six case studies of non-small-cell lung cancer in patients randomized to receive specific active immunotherapy in controlled clinical trials. Stewart con- cluded that dormancy in these patients is the result of immune mechanisms. Also in this review, animal models of tumor dormancy were discussed; again, it was stated that the evidence is clear that dormancy can be induced by manipulating immune mechanisms. Gray and Watkins, Jr. (1975) presented a general review of immunotherapy and stated that long-term tumor dormancy can be explained only by host defense mechanisms.

The effects of the immune system and how immune mechanisms could lead to oscillations in tumor size and to dormancy have also been modeled mathematically. In (de Boer and Hogeweg 1986), a mathematical model of the cellular immune response was used to investigate immune reactions to tumors. It was found that initially small doses of antigens do lead to tumor dormancy. The mathematical model of (Kirschner and Panetta 1998), which also focuses on the tumor-immune interaction, indicated that the dynamics between tumor cells, immune cells, and IL-2 can explain both short-term oscillations in tumor size as well as long-term tumor relapse. The mathematical model developed by Kuznetsov (Kuznetsov and Makalkin 1992, Kuznetsov et al. 1994), in which the nonlinear dynamics of immunogenic tumors are examined, also exhibits oscillatory growth patterns in tumors, as well as dormancy and "creeping through": when the tumor stays very small for a relatively long period of time, and subsequently grows to be dangerously large. In these mathematical models, the cyclical behavior of the tumor is directly attributable to the interaction with the immune response.

In models such as those of Kuznetsov et al. (1994) and Kirschner and Panetta (1998), immune cells and tumor cells compete in what is known as a "predator-prey" interaction, in which the immune cells play the role of the predator and the tumor cells play the role of the prey. This competition can give rise to cyclic growth and reduction in the cell populations in an intuitive way. The presence of tumor cells biochemically stimulates the production of immune cells. Simultaneously, the growth of the tumor cells is retarded by the presence of the immune cells. As the tumor cells die off, the immune cell population consequently decreases. But a decreasing immune cell pop- 


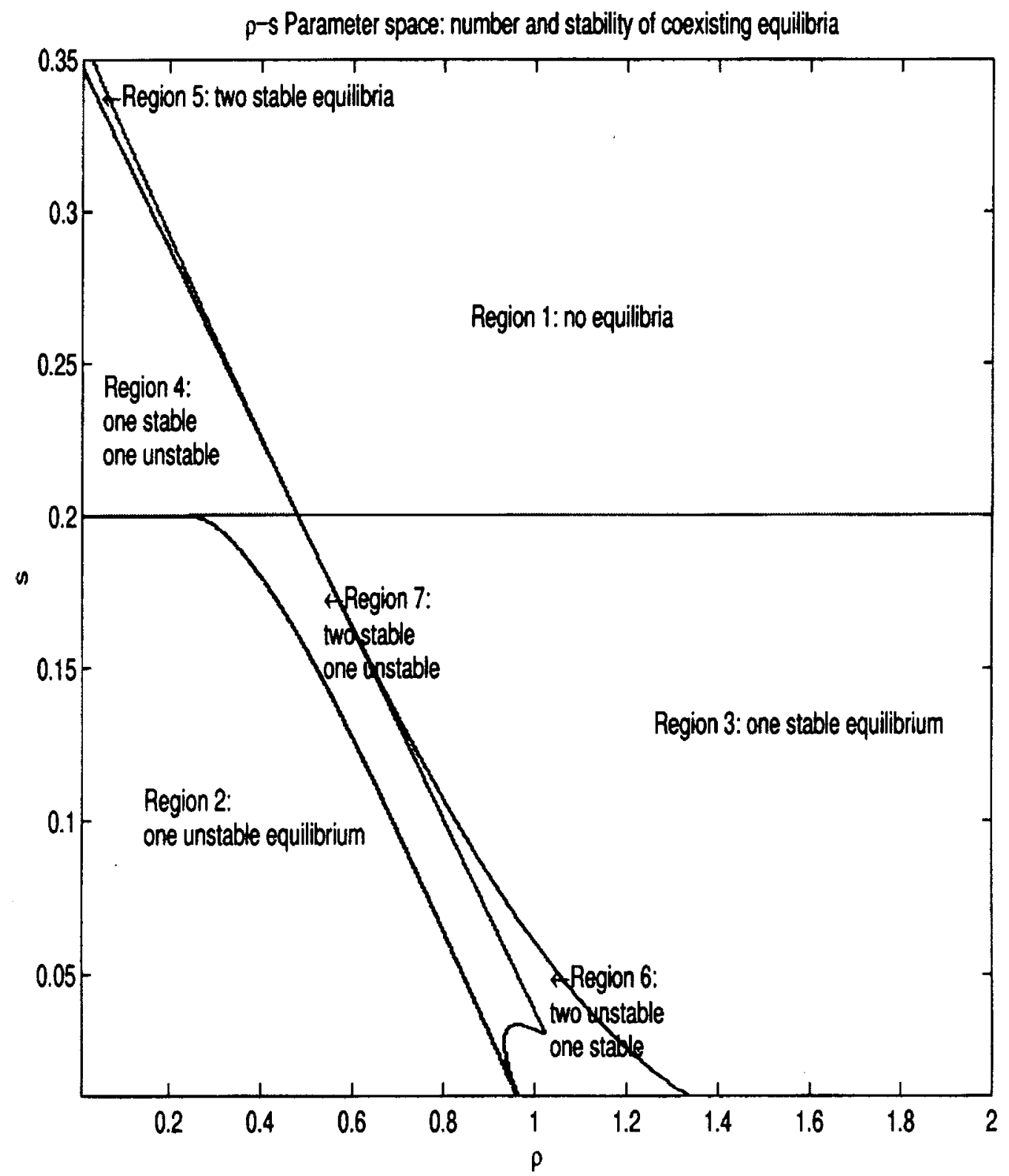

FIGURE 2 Coexisting equilibria as a function of immune response rate $\rho$ and source rate $s$. Depicted are Region 1: no equilibria, Region 2: one unstable equilibrium, Region 3: one stable equilibrium. Region 4: one stable and one unstable equilibrium, Region 5: two stable equilibria, Region 6: two unstable equilibria and one stable equilibrium, and Region 7: two stable equilibria and one unstable equilibrium (See Color Plate II at the back of this issue)

ulation will allow the tumor cells to begin growth once again. Depending on the system parameters, the cycle could continue indefinitely, or eventually spiral to a point of equilibrium. Because it is clear that the action of the immune cells significantly impacts the dynamics of tumor growth, we include the interaction of the immune and tumor cells in our model. In the model we develop, it is easily shown that if the 
immune system is removed, cyclical behavior cannot arise. This is because the resulting competitive system has either one globally stable equilibrium (stable competition) or two stable equilibria and a saddle point (competitive exclusion). See, for example, (Borrelli and Coleman 1998, p. 282) for a discussion of stable competition and competitive exclusion.

\subsection{Optimal Control Theory}

Once an adequate model of interacting cell populations is constructed, we then focus on the design of an improved treatment protocol. To this end, we employ the tools of optimal control theory. This theory originated in economics, where it was used to optimize returns on investments. It was subsequently applied to engineering problems and finally to biological models. The goal of chemotherapy is to destroy the tumor cells, while maintaining adequate amounts of healthy tissue. From a mathematical point of view, adequate destruction of tumor cells might mean forcing the system out of the basin of an unhealthy spiral node, or out of a limit cycle, and into the basin of attraction of a stable, tumor-free equilibrium. Alternatively, if the therapy pushes the system into a limit cycle in which the size of the tumor is small for a long period of time (as long as the life of the patient, for example), this could also be considered a "cure."

Optimality in treatment might be defined in a variety of ways. Some studies have been done in which the total amount of drug administered is minimized, or the number of tumor cells is minimized (Swierniak, Polanski, and Kimmel 1996), (Swierniak and Polanski 1994), (Swierniak 1994). The general goal is to keep the patient healthy while killing the tumor. Since our model takes into account the toxicity of the drug to all types of cells, we chose to minimize the tumor population, while constraining the normal cells to stay above some minimum level. Therefore, the development of a chemotherapy protocol can be phrased as an optimal control problem with constraints: for a fixed time interval, find the points within that interval at which the drug should be administered so that the number of tumor cells has been minimized, while the number of healthy cells has been kept above a prescribed threshold.

\subsection{Numerical Methods}

While general optimal control problems can often be difficult to solve analytically, one can sometimes appeal to numerical methods for obtaining solutions. Numerical methods for constrained optimal control are very sensitive to parameter adjustments, and do not always converge to realistic solutions, so in this arena we must exercise caution as well. We have employed a numerical approach to optimal control to determine a set of potentially optimal courses of treatment. A numerical approach has been used in, for example, (Martin 1992), (Martin et al. 1990), (Knolle 1988), (Murray 1990), (Swan 1987) and (Swan 1985), for simpler models without interaction between different cells. We present numerical results based on our model, and compare these solutions to a standard, periodically pulsed therapy.

\section{THE MODEL}

In this section we describe in detail the model we developed.

\subsection{The Model - Overview}

Culling useful aspects of previously developed mathematical models, we combine the following features in this model:

- Immune response: the model includes immune cells whose growth may be stimulated by the presence of the tumor and that can destroy tumor cells through a kinetic process. We point out that the presence of a detectable tumor in a system does not necessarily imply that the tumor has completely escaped active immunosurveillance. It is entirely possible that although a tumor is immunogenic, the immune system response is not sufficient on its own to completely combat the rapid growth of the tumor cell population and the even- 
tual development into a tumor. In fact, there is even some speculation that all tumors are immunogenic; see, for example, (Prehn 1994).

- Competition terms: normal cells and tumor cells compete for available resources, while immune cells and tumor cells compete in a predator-prey fashion.

- Optimal control theory for chemotherapy: a set of optimal drug therapies is calculated that minimize the tumor population by the end of the treatment period, while keeping the normal cells above a required level; these solutions are then used to design a practical treatment protocol.

We focus on tissue near the tumor site, and we assume a homogeneous tumor. We choose to model the reaction of the immune cells with the tumor cells in the same manner as that described in (Kuznetsov, Makalkin, Taylor and Perelson 1994). For the growth law terms, we considered several possible models, including exponential growth, Gompertz growth, and logistic growth. The exponential growth law in the context of a tumor cell population assumes that the rate of increase in the population at a certain point in time is directly proportional to the size of the tumor population at that time; the exponential curve is unbounded as time increases. The pattern of growth to which the Gompertz law gives rise is similar to that of exponential growth in the early stages, but plateaus as tumor size increases; the Gompertz growth curve is sigmoid. The logistic growth law is again similar to the exponential growth law, with the exception that it includes an intrinsic population carrying capacity beyond which the population size cannot grow. In cases in which specific biological data are available, the choice of growth law term and the parameters involved can be important. In (Vaidya and Alexandro, Jr. 1982), the derivation and behaviors of all three of the above growth laws, in addition to a fourth law, Bertalanffy growth, are described in detail. Each of these laws was evaluated against clinical data on untreated primary carcinoma of the human lung, as well as induced sarcoma in mice. The authors found that Bertalanffy growth gave the best results in the cases of mouse sarcoma, but that logistic growth most accurately described the progression of human lung carcinoma. In a more recent study (Hart, Shochat, and Agur 1998) the Gompertz, logistic, exponential, and power growth laws were compared. The power growth law is a direct generalization of exponential growth and is fully described in the study. In this case, model outcomes were compared to clinical data for primary breast cancer growth. For these particular breast cancer studies, the power growth law with an exponent of about 0.5 gave the best fit to the data.

Since the model we are developing is intended to be qualitative and does not focus on a particular tumor type, it is not immediately apparent how to measure which growth law is superior in this context. It turns out, however, that the growth law terms we compared allow for similar growth behavior up to a certain point in tumor size. Since we assume an initially small tumor mass, that is, a tumor size that is close to zero relative to carrying capacity, the choice of growth law does not significantly affect the qualitative behavior of the model. We compared the results of the evolution of our system using the various growth law terms and in each case found qualitative results to be similar. The solutions presented here, therefore, are those that have arisen using logistic growth. In Sections 5 and 6, we present analytic and numerical results of this new model, as well as open questions and future directions for refining the model.

Preliminary numerical results have already suggested that standard treatment protocols may not be optimal and that better outcomes may be achieved by administering medication in ways that have not been previously employed clinically but have been suggested by the mathematics. As this new model is developed and refined, these theories can be more thoroughly tested. Although there is still much to be done to test the new theories, every new result has the potential to be an advance towards improving the quality of treatment for cancer sufferers.

\subsection{The Model - Equations}

We let $I(t)$ denote the number of immune cells at time $t, T(t)$ the number of tumor cells at time $t$, and $N(t)$ the number of normal, or host cells at time $t$. The source 
of the immune cells is considered to be outside of the system, so it is reasonable to assume a constant influx rate, $s$. Furthermore, in the absence of any tumor, the cells will die off at a per capita rate $d_{1}$, resulting in a long-term population size of $s / d_{1}$ cells. Thus, immune cell proliferation will never suffer from immune upon immune crowding.

The presence of tumor cells stimulates the immune response, represented by the positive nonlinear growth term for the immune cells

$$
\frac{\rho I(t) T(t)}{\alpha+T(t)}
$$

where $\rho$ and $\alpha$ are positive constants. This type of response term is of the same form as the terms used in the respective models of Kuznetsov et al. (1994) and Kirschner and Panetta (1998). It is also similar to the one used by Owen and Sherratt (1998) once their system is reduced to the pseudo-steady state. In other words, as a function of $T$, it is positive, increasing, and concave.

Furthermore, the reaction of immune cells and tumor cells can result in either the death of tumor cells or the inactivation of the immune cells, resulting in the two competition terms

$$
\frac{d I}{d t}=-c_{1} I(t) T(t) \quad \text { and } \quad \frac{d T}{d t}=-c_{2} I(t) T(t) .
$$

As discussed in Section 2.1, the tumor cells as well as the normal cells are modeled by a logistic growth law, with parameters $r_{i}$ and $b_{i}$ representing the per capita growth rates and reciprocal carrying capacities of the two types of cells: $i=1$ identifies the parameters associated with the tumor, and $i=2$ identifies those associated with the normal tissue. In addition, there are two terms representing the competition between tumor and host cells.

Putting all the terms together gives the following system of ordinary differential equations:

$$
\begin{aligned}
\dot{I} & =s+\frac{\rho I T}{\alpha+T}-c_{1} I T-d_{1} I \\
\dot{T} & =r_{1} T\left(1-b_{1} T\right)-c_{2} I T-c_{3} T N \\
\dot{N} & =r_{2} N\left(1-b_{2} N\right)-c_{4} T N
\end{aligned}
$$

The behavior of this system without drug interactions will be analyzed in Section 3.

We now add the effect of the drug on the system. We denote by $u(t)$ the amount of drug at the tumor site at time $t$. We assume that the drug kills all types of cells, but that the kill rate differs for each type of cell, with the response curve in all cases given by an exponential

$$
F(u)=a\left(1-e^{-k u}\right)
$$

where $F(u)$ is the fraction cell kill for a given amount of drug, $u$, at the tumor site. Since the details of the pharmacokinetics are unknown, we let $k=1$ in these preliminary studies. We denote by $a_{1}, a_{2}$, and $a_{3}$ the three different response coefficients. We add these terms to the system of differential equations above as well as an equation for $u(t)$, the amount of drug at the tumor site. This is determined by the dose given, $v(t)$, and a per capita decay rate of the drug once it is injected. The system with drug interaction is then given by

$$
\begin{aligned}
\dot{I}= & s+\frac{\rho I T}{\alpha+T}-c_{1} I T-d_{1} I-a_{1}\left(1-e^{-u}\right) I \\
\dot{T}= & r_{1} T\left(1-b_{1} T\right)-c_{2} I T-c_{3} T N \\
& -a_{2}\left(1-e^{-u}\right) T \\
\dot{N}= & r_{2} N\left(1-b_{2} N\right)-c_{4} T N-a_{3}\left(1-e^{-u}\right) N \\
\dot{u}= & v(t)-d_{2} u
\end{aligned}
$$

Our control problem consists of determining the function $v(t)$ that will minimize the number of tumor cells at some specified time, $t_{f}$, with the constraint that we do not kill too many normal cells. If the units of cells are normalized, so that the carrying capacity of normal cells is 1 (i.e., $b_{2}=1$ ), we then require that the number of normal cells stay above three-fourths of the carrying capacity, or $N(t) \geq .75$ for all $t$. Therefore, in the language of optimal control theory, our objective function (the function we wish to minimize) and our constraint are given by

$$
\begin{array}{r}
\text { Objective Function: } J\left(t_{f}\right)=T\left(t_{f}\right) \\
\text { Constraint: } N(t) \geq .75,0 \leq t \leq t_{f}
\end{array}
$$

In Section 4 we look more closely at the optimal control problem and discuss some possible modifications to the objective function. 

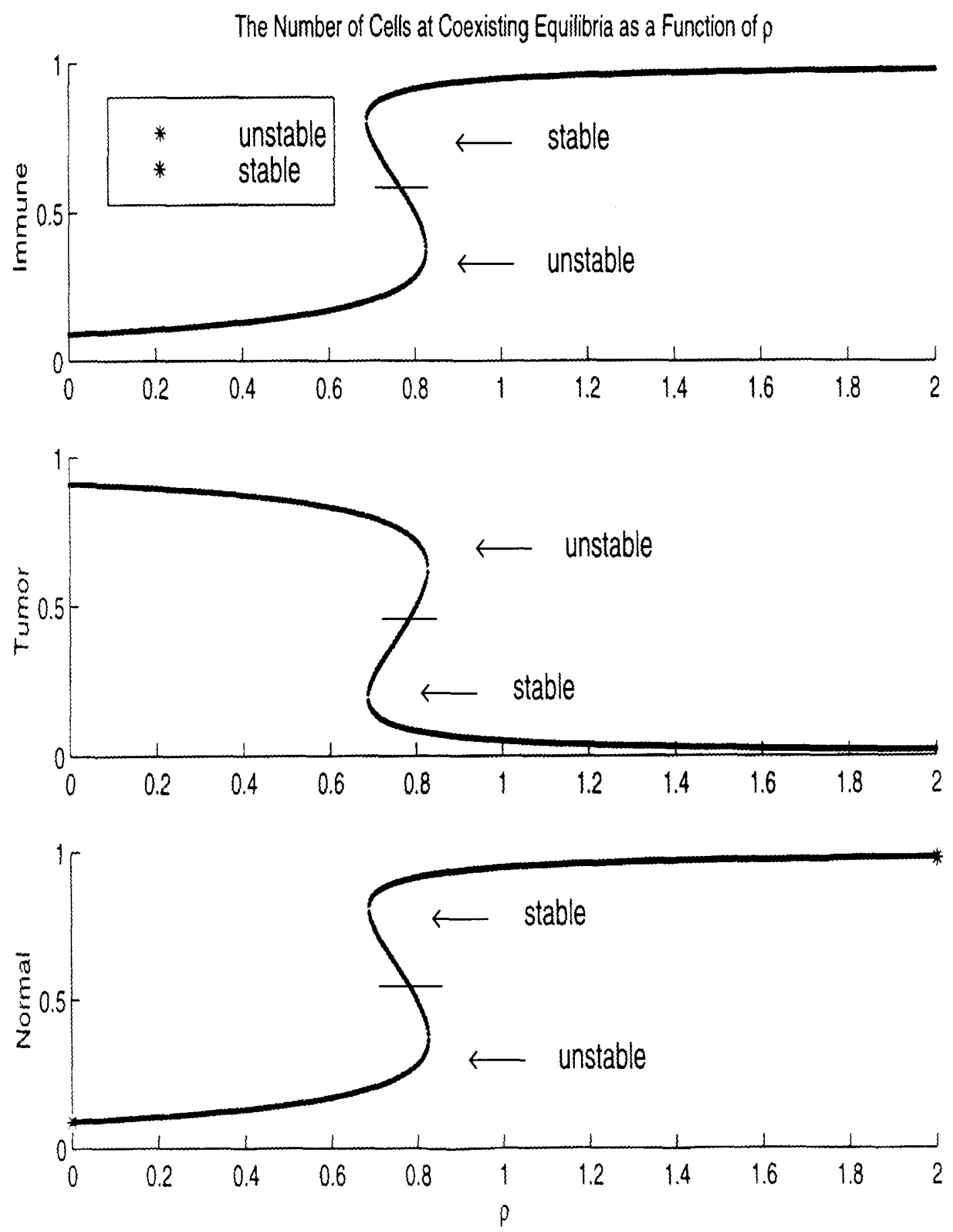

FIGURE 3 Cell populations at equilibria as a function of $\rho$. Stability of equilibria is indicated. Movement is from Region 2 through Regions 7 and 6 and finally into Region 3: as $\rho$ increase from 0.1 to 2.0 . Source rate $s=0.05$ (See Color Plate III at the back of this issue) 


\section{DRUG-FREE EQUILIBRIA}

To better understand the dynamics of the system, we first analyze the system without any drug input $(u(t)=0$ for all $t)$. Recall from Section 2 that the units of cells are normalized so that $b_{2}=1$. In order to consider the patient "cured," the system must be either in the basin of a stable tumor-free equilibrium or in the basin of a stable equilibrium at which only a harmlessly small amount of tumor is present.

The three sets of null-surfaces of the drug-free system given by (1) are described by the following:

- $N_{1}$ :

$$
\dot{I}=0 \Rightarrow I=\frac{s(\alpha+T)}{\left(c_{1} T+d_{1}\right)(\alpha+T)-\rho T}
$$

as long as $\rho T \neq\left(\mathrm{c}_{1} T+d_{1}\right)(\alpha+T)$.

$N_{1}$ is a curved cylindrical surface parallel to the $N$-axis. Letting $f(T)$ be a function of the tumor population $T$, we let $f(T)$ describe $N_{1}$ by defining

$$
f(T) \equiv \frac{s(\alpha+T)}{c_{1} T(\alpha+T)+d_{1}(\alpha+T)-\rho T}
$$

- $N_{2}$ :

$$
\dot{T}=0 \Rightarrow\left\{\begin{array}{l}
T=0 \\
\text { or } \\
T=1 / b_{1}-\left(c_{2} / r_{1} b_{1}\right) I-\left(c_{3} / r_{1} b_{1}\right) N
\end{array}\right.
$$

$N_{2}$ is a plane.

- $N_{3}$ :

$$
\dot{N}=0 \Rightarrow\left\{\begin{array}{l}
N=0 \\
\text { or } \\
N=1-\left(c_{4} / r_{2}\right) T
\end{array}\right.
$$

$N_{3}$ is also a plane, parallel to the $I$-axis. Letting $g(T)$ be a function describing $N_{3}$ in terms of the tumor population, we define

$$
g(T) \equiv 1-\left(c_{4} / r_{2}\right) T
$$

The null-surfaces for the particular set of parameter values used in our experiments are pictured in Figure 1. See Section 5.1 for a list of parameter values. The types of equilibrium points that could occur at the intersections of these surfaces can be classified as follows:
- Tumor-free: In this category, the tumor cell population is zero but the normal cells survive. The equilibrium point has the form

$$
\left(s / d_{i}, 0,1\right)
$$

- Dead: We classify an equilibrium point as "dead" if the normal cell population is zero. There are, therefore, two possible types of "dead" equilibria:

- Type-1: $\left(s / d_{1}, 0,0\right)$ in which both the normal and tumor cell populations have died off, and

- Type-2: $(f(a), a, 0)$ where the normal cells alone have died off and the tumor cells have survived. Here, $a$ is a nonnegative solution to

$$
a+\left(c_{2} / r_{1} b_{1}\right) f(a)-1 / b_{1}=0
$$

- Coexisting: Here, both normal and tumor cells coexist with nonzero populations. The equilibrium point would be given by

$$
(f(b), b, g(b))
$$

where $b$ is a nonnegative solution of

$$
b+\left(c_{2} / r_{1} b_{1}\right) f(b)+\left(c_{3} / r_{1} b_{1}\right) g(b)-1 / b_{1}=0
$$

Depending on the values of these parameters, there could be zero, one, two, or three of these equilibria. The two equilibrium states that the system should ideally approach, in the context of developing treatment therapy, are the tumor-free equilibrium and any coexisting equilibrium for which $b$ is small and $g(b)$ is close to 1 , since in these states the normal cell population survives.

\subsection{Tumor-Free Equilibrium}

In principle, we would like the tumor-free equilibrium to be stable so that the possibility exists of moving the state of the system toward the tumor-free point. In this section we discuss for which parameter ranges the tumor-free equilibrium is locally stable. Linearization around this equilibrium gives the system

$$
\left[\begin{array}{c}
\dot{I} \\
\dot{T} \\
\dot{N}
\end{array}\right]=\left[\begin{array}{ccc}
-d_{1} & \frac{\rho s}{d_{1} \alpha}-\frac{c_{1} s}{d_{1}} & 0 \\
0 & r_{1}-\frac{c_{2} s}{d_{1}}-c_{3} & 0 \\
0 & c_{4} & -r_{2}
\end{array}\right][I, T, N]^{T}
$$


with eigenvalues

$$
\begin{aligned}
& \lambda_{1}=-d_{1}<0 \\
& \lambda_{2}=r_{1}-\frac{c_{2} s}{d_{1}}-c_{3} \\
& \lambda_{3}=-r_{2}<0
\end{aligned}
$$

Thus the tumor-free equilibrium is stable as long as $\lambda_{2}<0$ or

$$
r_{1}<\frac{c_{2} s}{d_{1}}+c_{3}
$$

This relates the per-capita growth rate of the tumor cells, $\boldsymbol{r}_{1}$, to the "resistance coefficient," $c_{2} s / d_{1}$, which measures how efficiently the immune system competes with the tumor cells. If this tumor-free equilibrium is unstable, then according to this model, no amount of chemotherapy will be able to completely eradicate the tumor. This is in fact the case in the model of (Owen and Sherratt 1998) for all parameter values.

\subsection{Dead Equilibria}

The same type of analysis as above shows that the type- 1 dead equilibrium at $\left(s / d_{1}, 0,0\right)$ is always unstable. The type- 2 dead equilibrium at $(f(a), a, 0)$ can be either stable or unstable, depending on the parameters of the system. For any particular set of parameter values, one could apply the Routh test (see, for example, (Borrelli and Coleman 1998, p. 415)) to the characteristic polynomial of the Jacobian. For the parameter set used in our optimal control experiments, the type-2 dead equilibrium is located at $(2.85,0.05,0.0)$ and is unstable.

\subsection{Coexisting Equilibria}

Also of interest are the existence and stability of equilibria where a small tumor mass might coexist with a large number of normal cells. These equilibria occur at the intersection of the components of the three null-surfaces that do not correspond to coordinate planes. Figure 1 shows these surfaces, with the curved immune surface, $N_{1}$, depicted in the top graph, and the planar tumor and normal surfaces, $\mathrm{N}_{2}$ and $\mathrm{N}_{3}$, drawn on the same axes in the bottom graph. The three surfaces intersect at the coexisting equilibrium, which is marked with a dot on the graphs.

Depending on the parameter values, there can be zero, one, two, or three of these equilibria. The null-surfaces divide the positive octant into at most twelve regions. The goal of chemotherapy is to get the system into a region of stability of one of the "harmless" equilibria: either the tumor-free equilibrium at $\left(s / d_{1}, 0,1\right)$ or an equilibrium at which only a small amount of tumor is present.

Figure 2 shows the existence and stability of these equilibria as a function of the immune response rate, $\rho$, and the immune source rate, $s$. All other parameter values are set to be equal to those used in our later experiments. For our parameter values with $\rho=2.0$ and $s=0.1$, there is only one coexisting equilibrium, and it is stable. That is, our experimental parameter values place us in Region 3.

In the three graphs of Figure 3, the equilibrium values of the cell populations are plotted as a function of $\rho$, with $s$ fixed at $s=0.05$. In these plots, we see the transition from Region 2 (one unstable equilibrium) through Region 7 (two stable, one unstable equilibrium) and Region 6 (one stable, two unstable) and finally to Region 3 (one stable equilibrium point).

Note that the behavior of the system is very sensitive to the values of $\rho$, the tumor response rate, and to $s$, the steady source rate of immune cells.

\section{OPTIMAL THERAPY PROTOCOLS}

In this section we add the effect of chemotherapy treatments to our system, and we use optimal control theory to look for an optimal administration protocol.

Let

$$
h(I, T, N, u, v)=(\dot{I}, \dot{T}, \dot{N}, \dot{u})
$$

be the right-hand side of the system of differential equations describing the model. For brevity, we denote the state variables by $(I, T, N, u)=\left(x_{1}, x_{2}, x_{3}\right.$, $x_{4}$ ). We want to minimize the final number of tumor cells while keeping the normal cells above a fixed amount for the entire course of treatment. We have chosen this amount to be 75 percent of the tumor-free 

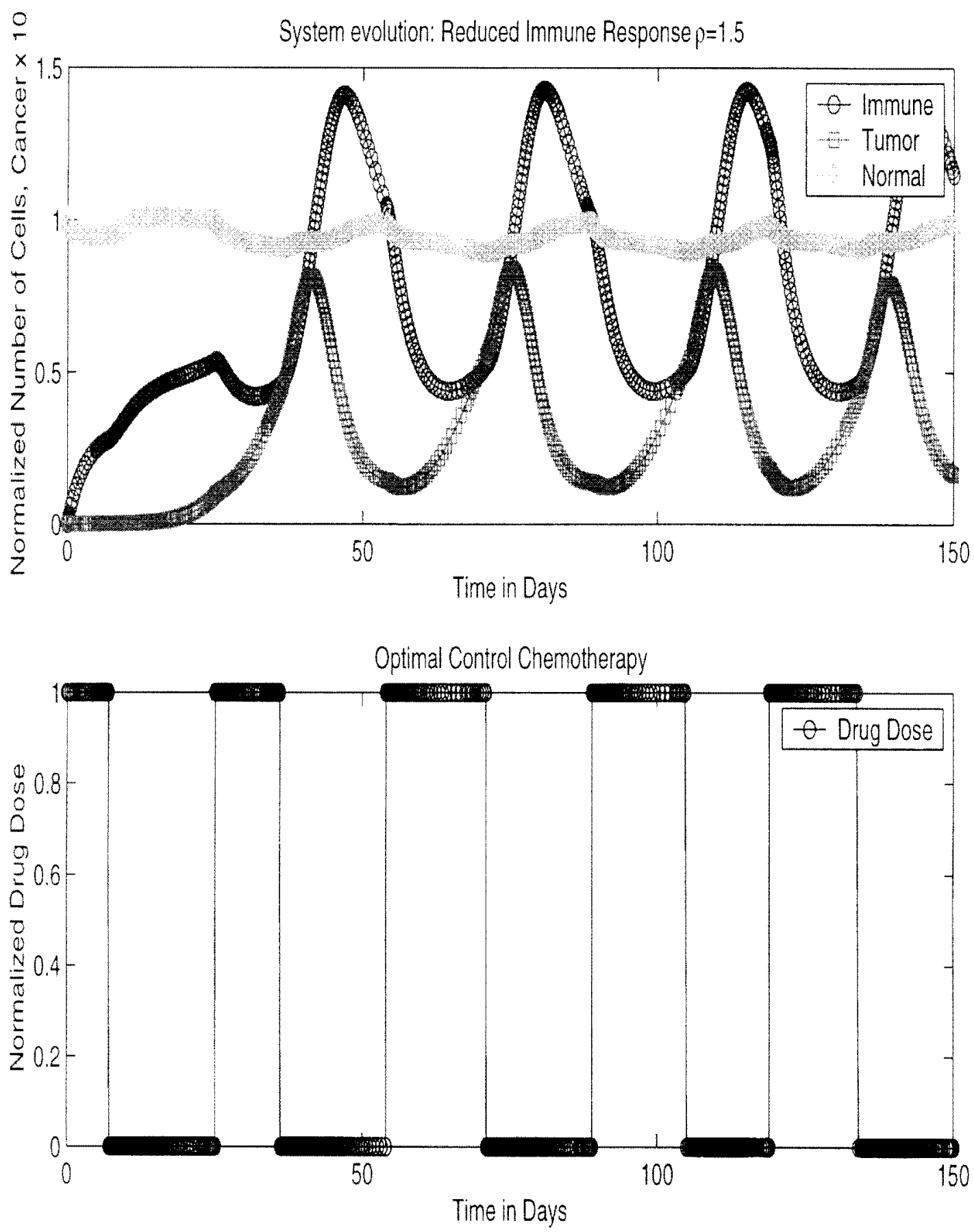

FIGURE 4 Optimal control solution with a lower value of $\rho$. Tumor cell population only is scaled up by a factor of 10 for visibility (See Color Plate IV at the back of this issue) 
normalized carrying capacity for this experiment. Thus, in the parlance of optimal control theory, the objective function is

$$
J(\mathbf{x}, v)=T\left(t_{f}\right)=x_{2}\left(t_{f}\right)
$$

and the inequality constraint is

$$
k(\mathbf{x}, t, v)=x_{3}(t)-.75 \geq 0 \quad 0 \leq t \leq t_{f}
$$

Using the standard approach (Kamien and Schwartz 1991), we derive the Hamiltonian for the optimal control problem:

$$
H=p_{1}\left(\dot{x}_{1}\right)+p_{2}\left(\dot{x}_{2}\right)+p_{3}\left(\dot{x}_{3}\right)+p_{4}\left(\dot{x}_{4}\right)+\eta k
$$

where the functions $p_{i}(t)$ satisfy the co-state equations, $\dot{p}_{i}=-\frac{\partial H}{\partial x_{i}}$ :

$$
\begin{aligned}
\dot{p}_{1}= & -p_{1}\left(\frac{\rho x_{2}}{\alpha+x_{2}}-c_{1} x_{2}-d_{1}-a_{1}\left(1-e^{-x_{4}}\right)\right) \\
& +p_{2} c_{2} x_{2} \\
\dot{p}_{2}= & -p_{1}\left(\frac{\rho \alpha x_{1}}{\left(\alpha+x_{2}\right)^{2}}-c_{1} x_{1}\right) \\
& -p_{2}\left(r_{1}-2 r_{1} b_{1} x_{2}-c_{2} x_{1}-c_{3} x_{3}-a_{2}\left(1-e^{-x_{4}}\right)\right) \\
& +p_{3} c_{4} x_{3} \\
\dot{p}_{3}= & p_{2} c_{3} x_{3} \\
& -p_{3}\left(r_{2}-2 r_{2} x_{3}^{2}-c_{4} x_{2}-a_{3}\left(1-e^{-x_{4}}\right)\right) \\
& -\eta(t) \\
\dot{p}_{4}= & -e^{-x_{4}}\left(a_{1} p_{1} x_{1}+a_{2} p_{2} x_{2}+a_{3} p_{3} x_{3}\right)
\end{aligned}
$$

and

$$
\eta(t) \geq 0 \quad \text { with } \quad \eta(t) k(t)=0
$$

Equation 9 along with the definition of $k$ in Equation 7 is consistent with

$$
\eta(t)= \begin{cases}1 & \text { for } x_{3}(t) \leq .75 \\ 0 & \text { otherwise }\end{cases}
$$

The boundary values for the co-state variables are given by $\left.\frac{\partial J}{\partial x_{i}}\right|_{t=t_{f}}$, or

$$
p_{1}\left(t_{f}\right)=0 ; p_{2}\left(t_{f}\right)=1 ; p_{3}\left(t_{f}\right)=0 ; p_{4}\left(t_{f}\right)=0
$$

The control equation is then

$$
\frac{\partial H}{\partial v}=p_{4}
$$

which is independent of the control variable, $v$. We assume that the amount of drug entering the patient at time $t$ is bounded above and satisfies

$$
0 \leq v(t) \leq v_{\max }
$$

We therefore have bang-bang solutions as candidates for optimal protocols; see (Kamien and Schwartz 1991):

$$
v= \begin{cases}0 & p_{4}>0 \\ v_{\max } & p_{4}<0 \\ \text { singular } & p_{4}=0\end{cases}
$$

Thus, the co-state variable, $p_{4}$, is the switching function for the system, and the drug should be injected at the maximum rate, $v_{\max }$, whenever $p_{4}$ is negative and should be stopped whenever $p_{4}$ is positive.

In the next section we describe some numerical solutions to this optimal control problem and compare them with a standard periodic protocol, where each treatment is of relatively short duration.

\section{NUMERICAL SOLUTIONS}

The problem as formulated in Section 4 is a two-point boundary value problem (TPBVP) for which the initial states of the state variables are known, and the final states of the co-state variables are known. For these numerical experiments we used a direct collocation method to solve the TPBVP, implemented in DIRCOL v1.2 (von Stryk 1999). The algorithm is sensitive to user input at various stages: in particular, it fails if the initial estimates of the state variables and the control variable are not close enough to optimal values. The grid of points at which the control is given is also crucial to the success of the algorithm.

\subsection{Parameters}

In this section we summarize the parameters of the model in lexicographic order. Since our model is qualitative, rather than quantitative, there is no claim that these values are fully realistic. While we attempt to use reasonable parameter values, there is still much work to be done toward accurate parameter-value estimation. In fact, since the model itself is still a pre- 

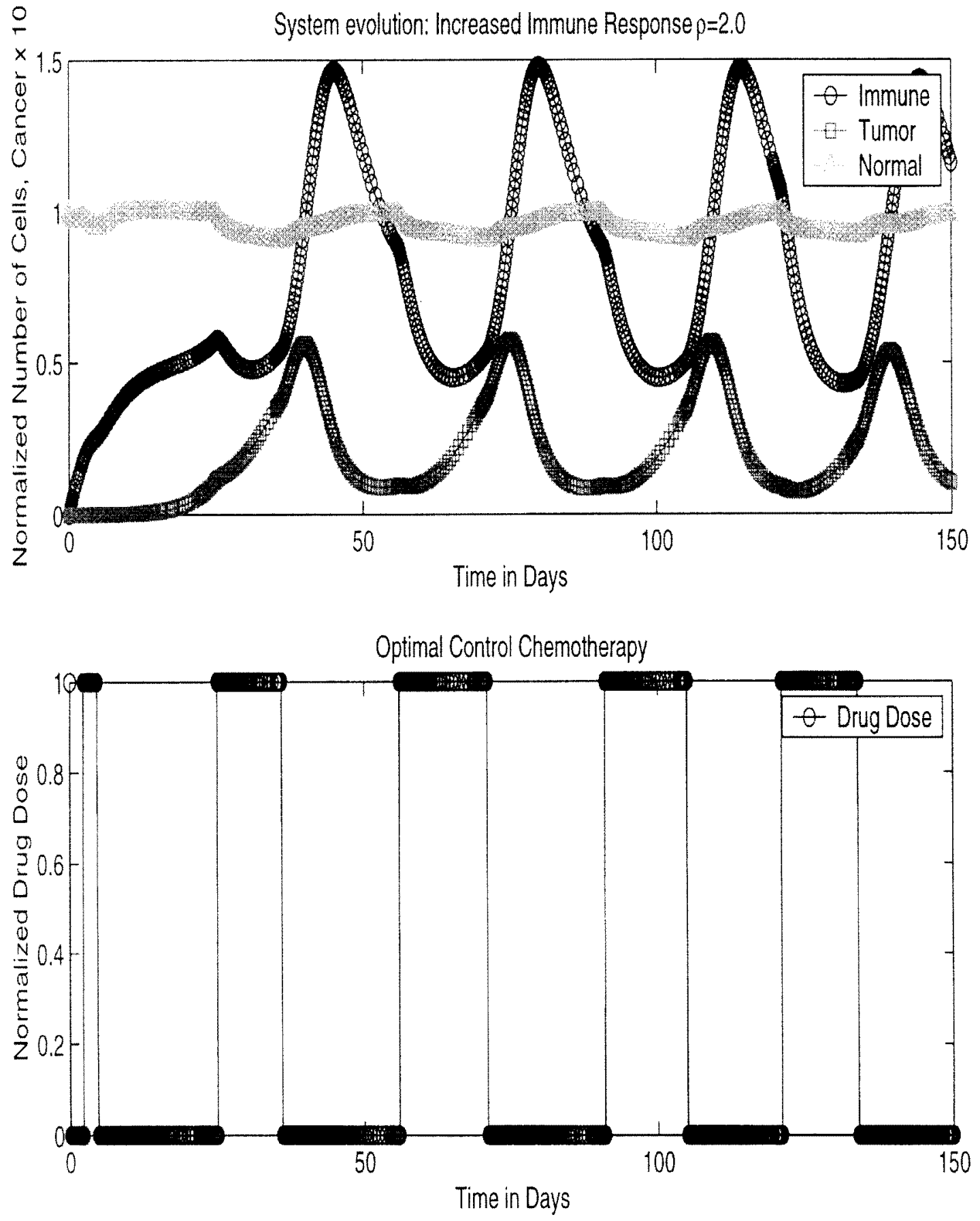

FIGURE 5 Optimal control solution with a higher value of $\rho$. Tumor cell population only is scaled up by a factor of 10 for visibility (See Color Plate V at the back of this issue) 
liminary one, we do not suggest that it produces quantitative results that reflect real-life quantities. Rather, we believe that the qualitative behavior of the model does indeed reflect the qualitative behavior of real tumors with respect to the response to treatment.

Recall that the units of cells were rescaled, so that one unit represents the carrying capacity of the normal cells in the region of the tumor. This depends on the type of tumor, of course, but it is reasonable to allow this to be on the order of $10^{11}$ cells (Rieker 1999). If one assumes that there are between $10^{8}$ and $10^{9}$ cells per cubic centimeter of tissue, then the normal cell population at carrying capacity encompasses a volume with a diameter somewhere between 5.8 and 12.4 centimeters. The parameter ranges implemented are as follows:

- Fraction Cell Kill: $0 \leq a_{i} \leq 0.5$, with $a_{3} \leq a_{1} \leq a_{2}$. In our experiments, these numbers were considered variable in the sense that different drugs provide for different cell kill rates. On the other hand, we wanted to avoid unreasonably efficient drugs, hence the upper bound of 0.5 on all the values.

- Carrying Capacities: $b_{1}^{-1} \leq b_{2}^{-1}=1$.

- Competition Terms: $c_{1}, c_{2}, c_{3}, c_{4}$ taken to be positive in these experiments. It is reasonable to assume that $c_{2}$ is larger than the rest, since the competition between immune cells and tumor cells is most detrimental to the tumor cells. Some authors argue that $c_{3}$ might be negative, and there is clinical evidence for this (Panetta 1996), (Michelson and Leith 1996). A negative competition coefficient in this case would imply that instead of the normal cells destructively competing with the tumor cells for resources and space, the presence of the normal cells would in fact stimulate further growth of the tumor cell population. In these preliminary experiments, however, we assume destructive competition. and we stick to the case $0<c_{3}<c_{2}$. When the coefficient $c_{2}$ is greater that $c_{3}$, this simply indicates that the presence of the immune systen is more damaging to the tumor cell population than is the competition between the tumor cells and normal cells.
- Death Rates: $d_{1}$ and $d_{2}$. Here $d_{1}$ is the per capita death rate of the immune cells, with $d_{1}=.2$, and $d_{2}$ is the per capita death rate of the drug, with $d_{2}=1$.

- Per Unit Growth Rates: $r_{1}$ and $r_{2}$, with time normalized so that $r_{2}=1$. Depending on the type of cancer and the stage of growth, $r_{1}$ may be bigger or smaller that $r_{2}$. See, for example, (Kusama et al. 1972), (Arnerøv et al. 1992), and (Steel 1977). Here we assume that the tumor cell population grows more rapidly than the normal cell population, and let $r_{1}>r_{2}$.

- Immune Source Rate: $s$, a steady source rate for immune cells in the absence of a tumor. In our experiments, $0 \leq s \leq .5$; see (Kuznetsov et al. 1994).

- Immune Threshold Rate: $\alpha$, which is inversely related to the steepness of the immune response curve. When the number of tumor cells, $T$, is equal to $\alpha$, the immune response rate is at half of its maximum value. We used $\alpha=3$. See, for example, the parameter estimation work in (Kuznetsov et al. 1994).

- Immune Response Rate: $\rho$, which we assume to have a baseline value of 1 . With the other parameter choices, an interesting range of $\rho$ is the interval $(0,2.5)$. In numerical experiments, we varied $\rho$ in this range to determine bifurcations in the behavior of the system of equations 2. See Figures 2 and 3 for illustrations of the effects of varying $\rho$.

Initial values are $I(0)=s / d_{1}, T(0)=10^{-5}, N(0)=1$. When chemotherapy is initiated, the initial tumor mass is small, and immune and normal cells are at their healthy equilibrium levels. We are assuming a situation in which as much of the tumor has been removed as is possible by surgery or radiation. An initial tumor population of $10^{-5}$ normalized units is equivalent to $10^{6}$ tumor cells. If these tumor cells formed a sphere, it would occupy a volume of radius between 0.12 and 0.27 centimeters. The clinical detection threshold for a tumor is generally $10^{7}$ cells (Shochat, Hart, and Agur 1999), so the initial tumor volume of $10^{-5}$ normalized units is below clinical detection levels. Note that the presence of a preopera- 


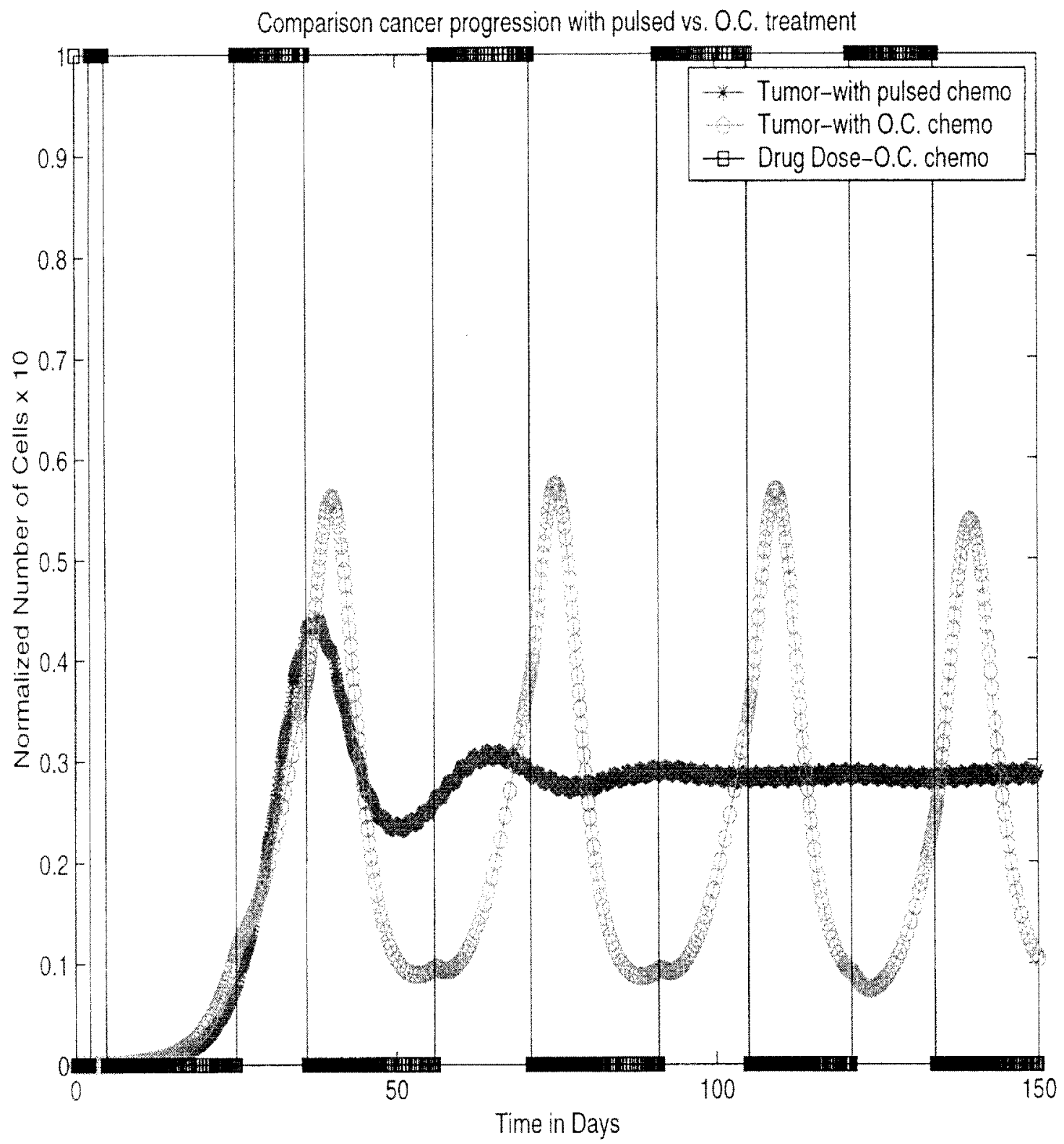

FIGURE 6 Comparison of optimal protocol with a standard pulsed protocol. Depicted is the optimal control protocol, as well as the comparison of the progression of the tumor in response to the optimal control protocol, versus the tumor response to pulsed protocol. The standard pulsed protocol, which is administered every other day for 12 hours, is implicit to the solution of the tumor response to traditional protocol but is not explicitly depicted on the graph. The normalized tumor cell population sizes are scaled up by a factor of ten on the plot to enhance clarity (See Color Plate VI at the back of this issue) 
tive clinically detectable tumor does not necessarily imply that the tumor has completely escaped immunosurveillance, simply that the immune system response was not sufficient to curtail the rapid growth of the tumor cell population.

Results from preliminary numerical experiments follow. Figures 4 and 5 show typical optimal solutions. The upper graphs show the time evolution of the three types of cells, while the lower graphs show the control variable, $v$. Notice that the numerical simulation did produce bang-bang type optimal solutions. Comparison of the two graphs shows the effect of changing the parameter $\rho$, showing greater tumor reduction in the case of higher immune response rate. Since the tumor population is an order of magnitude smaller than the other populations, the amount of tumor on each of the plots has been scaled up by a factor of ten in order to make the difference in tumor progression visible. Note also that the control in the reduced immune response case calls for about $19 \%$ more total medication to be administered over the course of treatment.

\subsection{Comparison with Standard Protocols}

The protocol suggested by the optimal control algorithm dictates that the drug be administered continuously over relatively long periods of time-on the order of days. Standard protocol is to administer the drug for a short time, on the order of several hours, with periodically repeated treatments every few weeks. Figure 6 compares the optimal control protocol with a standard protocol of the periodically pulsed type. Although traditional pulsed chemotherapy is generally administered once every two or three weeks, in our experiments we increase the frequency of the pulses to every other day. This is to ensure that the total dose administered over the treatment period of 150 days is equivalent to the total dose administered with the optimal control protocol. Because of their high time frequency, the pulsed doses would obscure the graph and are therefore not depicted. However, the progression of the tumor in response to the pulsed doses is depicted. Note that the optimal control protocol allows the tumor to oscillate in size with larger amplitude, although it does result in a smaller tumor mass at the prescribed final time, $t_{f}$. Clinically it is clearly not considered desirable to induce such oscillations. However, it is important to keep in mind that our specific goal in the context of the optimal control problem is to minimize the final tumor size while keeping the patient healthy by some measure. The measure of health that we specify is in terms of the population of normal cells, which we require to stay above a certain minimum. The optimal control algorithm did exactly what it was directed to do, and did in fact reduce the final tumor mass with respect to the mass resulting from pulsed therapy, without allowing the normal cell population to oscillate by more than about $5 \%$. From Figures 4 and 5 it is clearly seen that there are only very small amplitude oscillations in the normal cell population.

We also compare the output of our model with data from (Thomlinson 1982) from a patient with a breast carcinoma. Figure 9, page 490 of (Thomlinson 1982) shows the progression of the size of a breast carcinoma and its response to injections of a combination of cytotoxic drugs. Thomlinson notes that tumor growth cycles are asynchronously offset from treatment times, making it appear that the patient could be resistant to the therapy, However, Thomlinson argues that drug resistance does not completely explain the asynchronous behavior. The same asynchronous phenomenon appears in our model, both with optimal control therapy and with traditional pulsed therapy. In this model, this behavior is caused by the detrimental effect of chemotherapy on the immune cells, and the subsequent interaction of the immune cells with tumor cells. This type of oscillatory behavior is in fact typical of many predator-prey interaction models. The results of the numerical experiment are shown in Figures 7 and 8 . Note that the tumor populations have been scaled up on the plots for clarity.

\section{DIRECTIONS FOR FUTURE WORK}

A natural extension of this work would be to study other objective functions in the optimal control problem, as described in Section 1.3. For example, we 


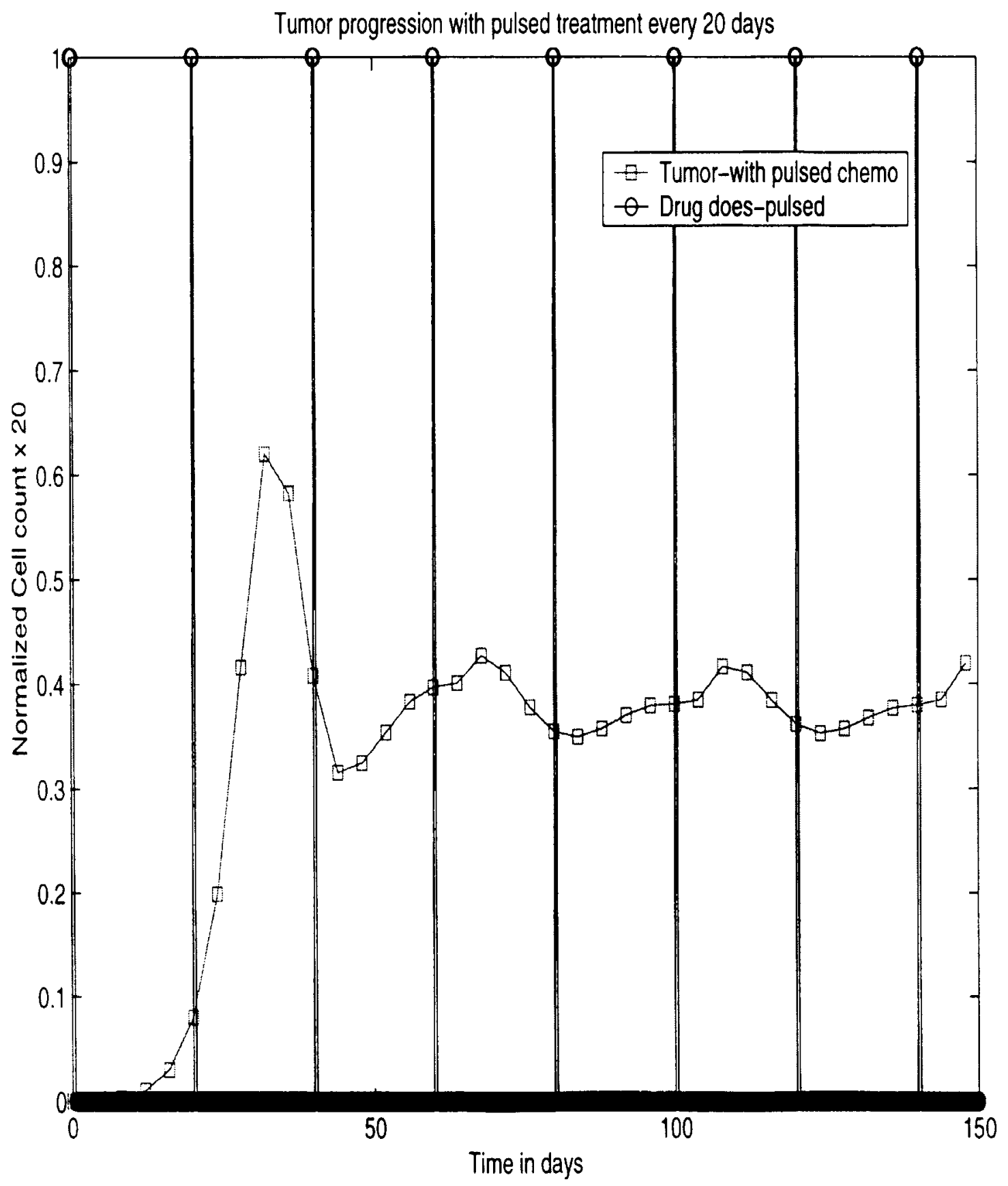

FIGURE 7 Oscillations in tumor size are asynchronous with pulsed chemotherapy. Tumor cell population is scaled up by a factor of 20 (See Color Plate VII at the back of this issue) 
might attempt to minimize a linear combination of the average tumor size and the final tumor size by letting

$$
J(\mathrm{x}, v, t)=K_{1} \frac{1}{t_{f}} \int_{0}^{t_{f}} T(t) d t+K_{2} T\left(t_{f}\right),
$$

where $K_{1}$ and $K_{2}$ are prioritizing weights. Note that this objective function reduces to that of Equation (3) if $K_{1}=0$ and $K_{2}=1$. This more generalized objective function might allow us to reduce the tumor size oscillations that appear with our current objective function. Alternatively, we may consider allowing the total time of treatment to vary. As we saw in Section 3 , for some parameter values there are coexisting equilibria, that is, equilibria at which all types of cells have positive values. In the case where such an equilibrium occurs at a small level of tumor cells but at a large level of normal cells, this point could represent a permanent "indolent" tumor. If the equilibrium were stable, a therapy that put the system in the basin of attraction of this indolent equilibrium would be considered a "cure." Thus our objective function might minimize the distance to this equilibrium, rather than the distance to the immune-normal cell plane (as is the case when the number of tumor cells is minimized).

An enhancement of the current model would take into account the cell cycle. We would begin by modeling the cell cycle of the tumor cells in two stages, where the drug affects the cell only in the mitotic stage. This would turn the differential equations into delay-differential equations and would complicate the optimal control problem but would not necessarily make it intractable. In fact, such problems have been extensively studied in applications to economics and management (Kamien and Schwartz 1991) where necessary conditions for optimality can sometimes be derived.

Another element of the model we wish to examine more closely is the assumption that the competition between cells, specifically the competition between tumor cells and normal cells, is in proportion to the product of their numbers. This assumes that each cell is equally likely to compete with each cell of the other type. While this assumption may be reasonable if we are dealing with liquid cancers, such as leukemia, in a solid tumor, such as breast cancer, the competition between the tumor and normal cells for resources is more likely to occur along the interface between the two. We therefore propose to look at a model that takes into account the geometry of the tumor and uses a stochastic, nearest-neighbor competition paradigm. The competition between the immune cells and the tumor cells could stay as it is, since it is based on cell interactions as described in Section 1.

Another refinement of the model would include time-varying competition terms. It is known among clinicians and through in vitro experiments that small tumors are inhibited by the presence of normal cells but that large tumors are stimulated by normal cells. In particular, there is evidence that the production of fibroblasts can stimulate tumor cell growth. We plan to incorporate this interaction into our model and study the resulting parameter space, focusing on the competition parameter $c_{3}$.

In clinical experience, a patient will respond at first to chemotherapy, and then cease to do so. One explanation for these symptoms is the evolution of drug-resistant subpopulations of tumor cells. We also plan to add this new population as another state variable to investigate the dynamical ramifications. Choosing a therapy would then involve also determining the time when the patient should be given a new batch of drugs. The statement of the optimal control problem would then change as well, with a new term being added to the objective function and to the control variable. The times at which drug combinations should be changed as well as the periods of drug administration would be chosen to minimize the new objective function.

\section{Acknowledgements}

We thank the members of the Mathematics Of Medicine group at St. Vincent's Hospital in Los Angeles, especially Dr. Charles Wiseman and Dr. Torn Starbird. We also thank Dr. Jeff Rieker of Pomona Valley Hospital for helpful discussions. The first author thanks Argonne National Laboratory and Harvey Mudd College for supporting this research. Argonne support is under U.S. Department of Encrgy Contract W-31-109-ENG-38. 


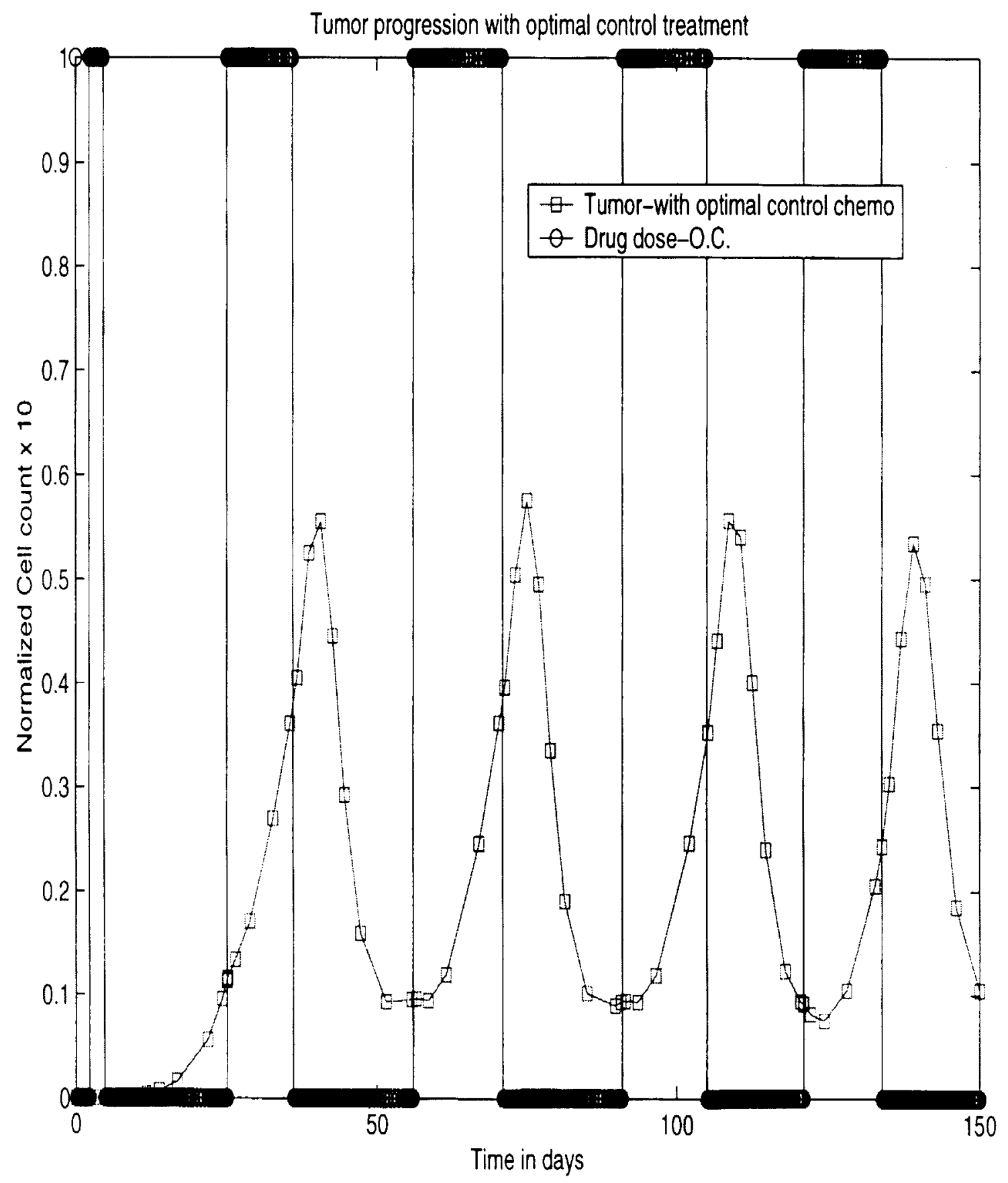

FIGURE 8 Oscillations in tumor size are asynchronous with optimal control chemotherapy. Tumor cell population is scaled up by a factor of 10 (See Color Plate VIII at the back of this issue) 


\section{References}

Adam, J. A.: 1993. The dynamics of growth-factor-modified immune response to cancer growth: One-dimensional models, Mathematical and Computer Modelling 17(3), 83-106.

Adam, J. A. and Panetta, J.: 1995, A simple mathematical model and alternative paradigm for certain chemotherapeutic regimens, Mathematical and Computer Modelling 22(8), 49-60.

Arnerøv, C., Emdin, S., Lundgren, B., Roos, G., Søderstrøm, I., Bjersing, L., Norberg, C. and Ângquist, K.: 1992, Breast carcinoma growth rate described by mammographic doubling time and S-phase fraction: Correlations to clinical and histopathologic factors in a screened population, Cancer 70(7), 19281934.

Bartońszyński. R., Jones, B. F. and Klein, J. P.: 1985, Some stochastic models of cancer metastases, Communications in Statistics. Stochastic Models 1(3), 317-339.

Borrelli, R, and Coleman, C.: 1998, Differential equations: A modeling perspective, John Wiley and Sons.

Bramson, M. and Griffeath, D.: 1980, The asymptotic behavior of a probabilistic model for tumor growth, Biological growth and spread (Proc. Conf., Heidelberg, 1979), Springer, Berlin, pp. $165-172$.

Castellanos Moreno, A.: 1996, A stochastic model for the evolution of cancerous tumors, Revista Mexicana de Física 42(2), 236249.

Coldman, A. J. and Goldie, J. H. : 1986, A stochastic model for the origin and treatment of tumors containing drug-resistant cells, Bulletin of Mathematical Biology 48(3-4), 279-292. Simulation in cancer research (Durham, N.C., 1986).

de Boer, R. and Hogeweg, P.: 1986, Interactions between macrophages and T-lymphocytes: tumor sneaking through intrinsic to helper $\mathbf{T}$ cell dynamics, Journal of Theoretical Biology 120(3), 331-51.

Dibrov, B.F., Zhabotinsky, A.M., Neyfakh, Y.A., Orlova, M.P. and Churikova, L. I.: 1985, Mathematical model of cancer chemotherapy. Periodic schedules of phase-specific cytotoxic-agent administration increasing the selectivity of therapy, Mathematical Biosciences. An nternational Journal 73(1), 1-31.

Duc, H. N.: 1985. A stochastic model of mutant growth due to mutation in tumors, based on stem cell considerations, Mathe matical Biosciences. An International Journal 74(1), 23-35.

Eisen, M.: 1979, Mathematical models in cell biology and cancer chemotherapy, Springer-Verlag, Berlin.

Farrar, J., Katz, K., Windsor, J., Thrush, G., Scheuermann, R., Uhr, J. Thrush G. Scheuermann R. Uhr J. and Street, N.: 1999, Cancer dormancy. VII. a regulatory role for CD8+ $\mathbf{T}$ cells and IFN-gamma in establishing and maintaining the tumor-dormant state, Journal of Immunology 162(5), 2842-9.

Gray, B. and Watkins Jr., E.: 1975, Immunologic approach to cancer therapy, Medical Clinics of North America 59(2), 327-37.

Hart, D., Shochat, E. and Agur, Z.: 1998, The growth law of primary breast cancer as inferred from mammography screening trials data, British Joumal of Cancer 78(3), 382-387.

Kamien, M. and Schwartz, N.: 1991, Dynamic Optimization: The Calculus of Variations and Optimal Control in Economics and Management, Vol. 31 of Advanced Textbooks in Economics, 2 edn, North-Holland.

Kirschner, D. and Panetta, J.: 1998, Modeling immunotherapy of the tumor-immune interaction, Journal of Mathematical Biology 37(3), 235-52

Knolle, H.: 1988, Cell kinetic modelling and the chemotherapy of cancer, Springer-Verlag, Berlin.
Kusama, S., Spratt. J., Donegan. W., Watson, F. and Cunningham, C.: 1972 , The gross rates of growth of human mammary carcinoma, Cancer 30(2), 594-599.

Kuznetsov, V. and Makalkin, I.: 1992, Bifurcation-analysis of mathematical-model of interactions between cytotoxic lymphocytes and tumor-cells - effect of immunological amplification of tumor-growth and its connection with other phenomena of oncoimmunology, Biofizika 37(6), 1063-70.

Kuznetsov, V., Makalkin, I., Taylor, M. and Perelson, A.: 1994, Nonlinear dynamics of immunogenic tumors: Parameter estimation and global bifurcation analysis, Bull. of Math. Bio. 56(2), 295-321.

Martin, R. B.: 1992, Optimal control drug scheduling of cancer chemotherapy, Automatica. The Journal of IFAC, the International Federation of Automatic Control 28(6), 1113-1123.

Martin. R. B., Fisher. M. E., Minchin, R. F. and Teo, K. L.: 1990, A mathematical model of cancer chemotherapy with an optimal selection of parameters, Mathematical Biosciences. An International Journal 99(2), 205-230.

Matsuzawa, A., Kaneko, T., Takeda, Y. and Ozawa, H.: 1991a, Characterization of a strongly immunogenic mouse leukemia line (DL811) undergoing spontaneous cure with tumor dormancy, Cancer Letters 58(1-2), 69-74

Matsuzawa, A., Takeda, Y., Narita, M. and Ozawa, H.: 1991b, Survival of leukemic cells in a dormant state following cyclophosphamide-induced cure of strongly immunogenic mouse leukemia (DL811), International Journal of Cancer 49(2), 303-9.

Michelson. S. and Leith, J.: 1996, Host response in turnor growth and progression, Invasion and Metastasis 16(4-5), 235-246.

Morecki, S., Pugatsch, T., Levi. S., Moshel, Y. and Slavin, S.: 1996 Tumor-cell vaccination induces tumor dormancy in a murine model of B-cell leukemia/ymphoma (BCL1), International Joumal of Cancer 65(2), 204-8.

Muller, M., Gounari, F., Prifti, S., Hacker, H., Schirrmacher, V. and Khazaie, K: 1998, EblacZ tumor dormancy in bone marrow and lymph nodes: active control of proliferating tumor cells by CD8+ immune T cells, Cancer Research 58(23), 5439-46.

Murray, J. M.: 1990, Optimal control for a cancer chemotherapy problem with general growth and loss functions, Mathematical Biosciences. An International Journal 98(2), 273-287.

Owen, M. and Sherratt, J.: 1998, Modelling the macrophage invasion of tumours: Effects on growth and composition, IMA Journal of Mathematics Applied in Medicine and Biology 15, $165-185$.

Panetta, J.: 1996, A mathematical model of periodically pulsed chemotherapy Tumor recurrence and metastasis in a competitive environment, Bulletin of Mathematical Biology 58(3), 425-447.

Prehn, R.: 1994, Stimulatory effects of immune-reactions upon the growths of untransplanted tumors, Cancer Research 54(4), 908-914.

Rieker, J.: 1999, Conversations. Physician with Pomona Valley Hospital, Pomona, California.

Serio, G.: 1984, Two-stage stochastic model for carcinogenesis with time-dependent parameters, Statistics \& Probability Letters 2(2), 95-103.

Shochat, E., Hart, D. and Agur, Z.: 1999, Using computer simulations for evaluating the efficacy of breast cancer chemotherapy protocols, Mathematical Models and Methods in Applied Sciences 9(4), 599-615.

Steel. G.: 1977. Growth kinetics of tumors, Oxford University Press, Oxford. 
Stewart, T.: 1996, Immune mechanisms and tumor dormancy, Medicina (Buenos Aires) 56(1), 74-82.

Swan, G. W.: 1985, Optimal control applications in the chemotherapy of multiple myeloma, IMA Journal of Mathematics Applied in Medicine and Biology 2(3), 139-160.

Swan, G. W.: 1987, Optimal control analysis of a cancer chemotherapy problem, IMA (Institute of Mathematics and its Applications). Journal of Mathematics Applied in Medicine and Biology 4(2), 171-184.

Swierniak, A.: 1994, Some control problems for simplest differential models of proliferation cycle, Applied Mathematics and Computer Science 4(2), 223-232.

Swierniak, A. and Polanski, A.: 1994, Irregularity in scheduling of cancer chemotherapy, Applied Mathematics and Compuler Science 4(2), 263-271.
Swierniak, A., Polanski, A. and Kimmel, M.: 1996, Optimal control problems arising in cell-cycle-specific cancer chemotherapy, Journal of Cell Proliferation 29, 117-139.

Thomlinson, R.: 1982, Measurement and management of carcinoma of the breast. Clinical Radiology 33(5), 481-493.

Vaidya, V. and Alexandro Jr., F.: 1982, Evaluation of some mathematical models for tumor growth, International Joumal of Bio-Medical Computing 13, 19-35.

von Stryk, O.: 1999, User's guide for DIRCOL: A direct collocation method for the numerical solution of optimal control problems, Lehrstuhl M2 Numerische Mathematik, Technische Universitaet Muenchen. Copyright (C) 1994-1999 Technische Universitaet Muenchen. 


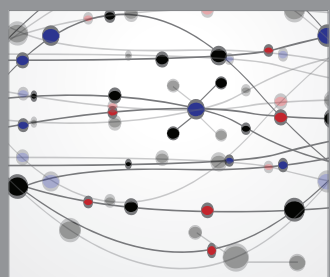

The Scientific World Journal
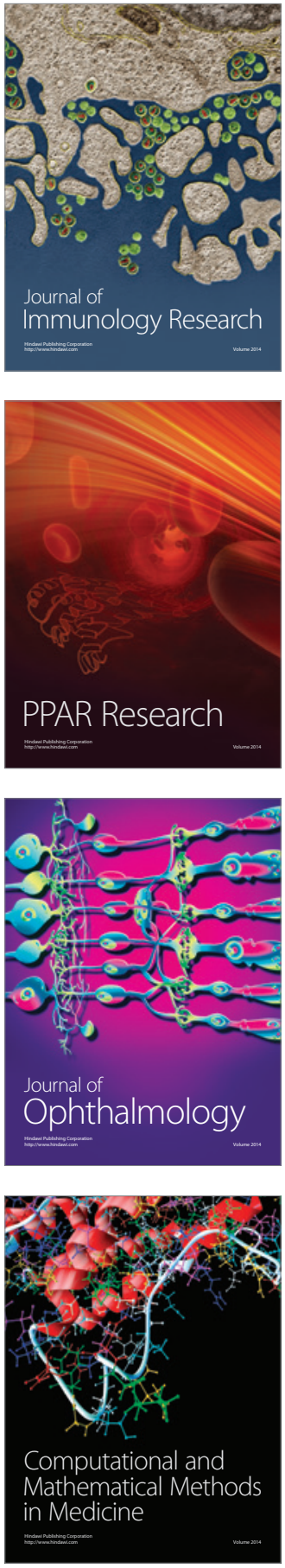

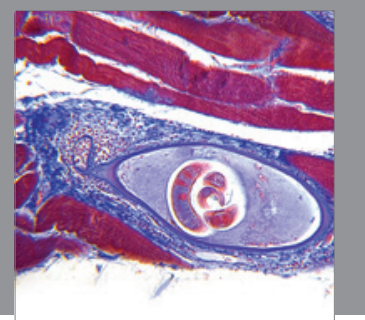

Gastroenterology

Research and Practice
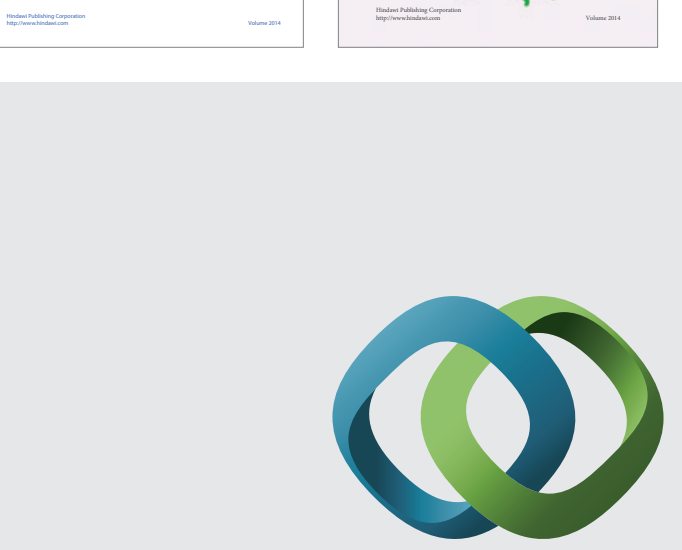

\section{Hindawi}

Submit your manuscripts at

http://www.hindawi.com
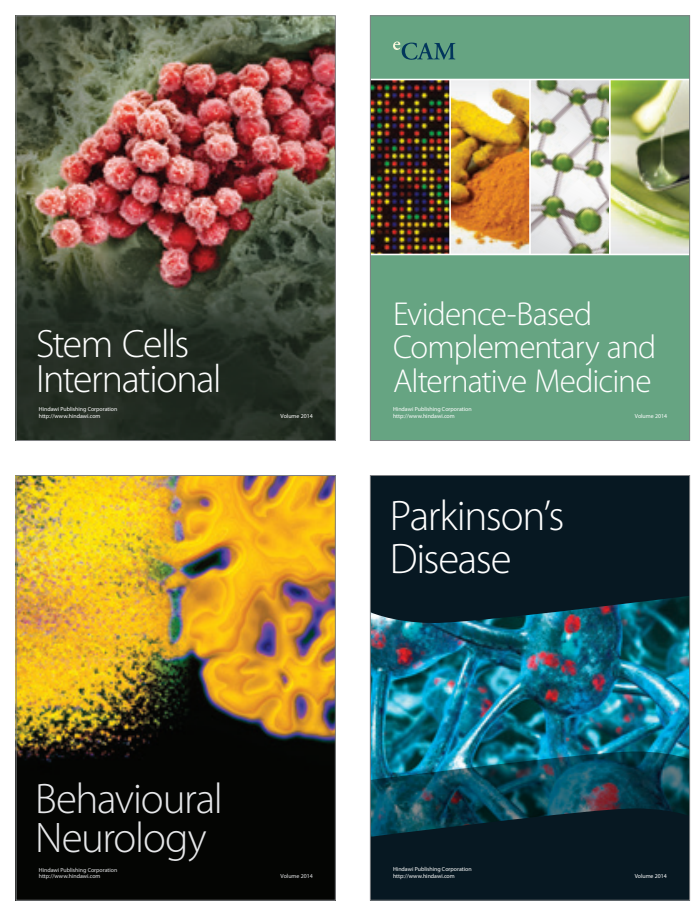

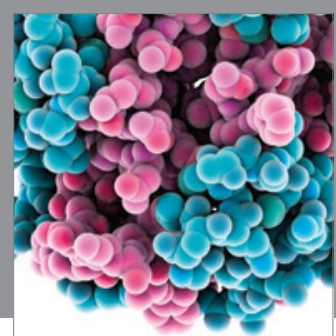

Journal of
Diabetes Research

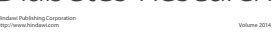

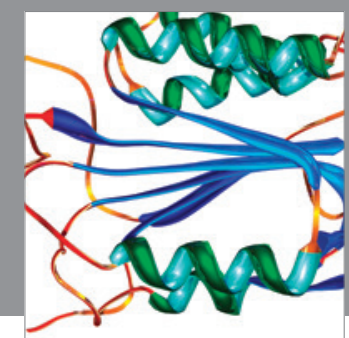

Disease Markers
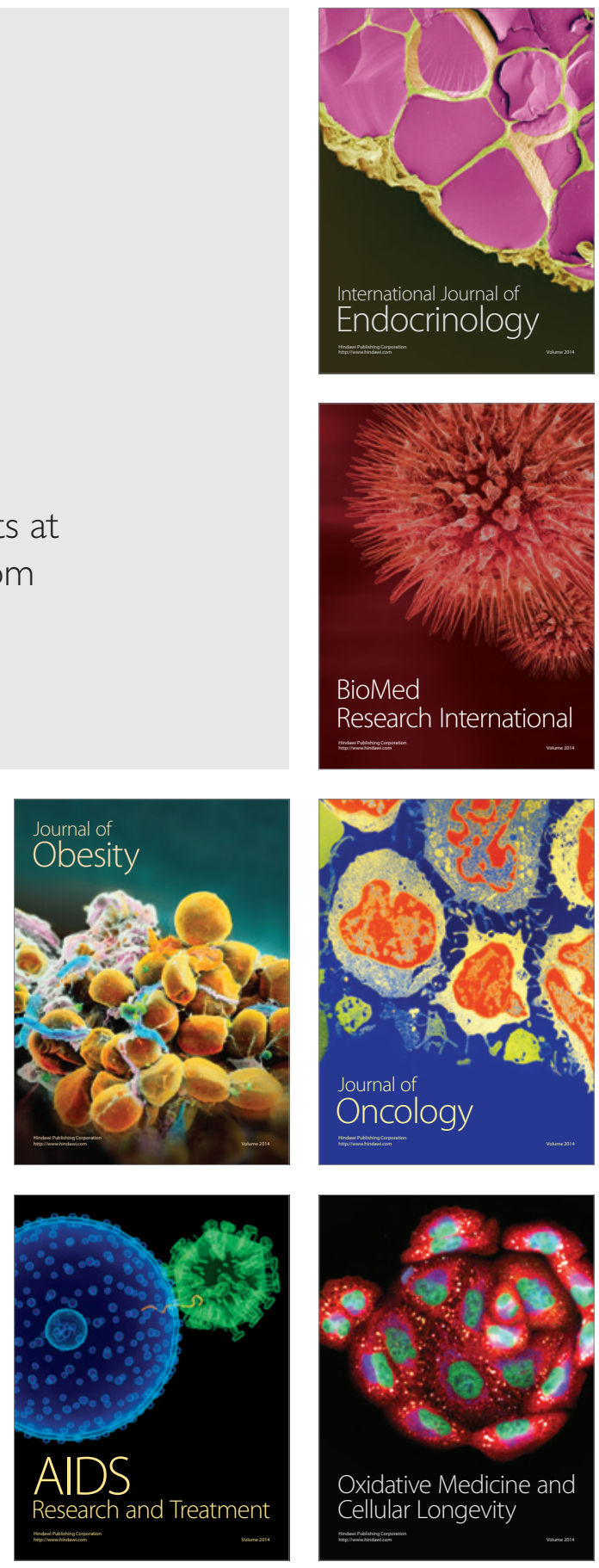